\title{
Food Addiction and Intermittent Fasting
}

PD Gupta

Centre for Cellular and Molecular Biology, Hyderabad, India
*Corresponding author

PD Gupta, Centre for Cellular and Molecular Biology, Hyderabad, India

Submitted: 24 Feb 2020; Accepted: 03 Mar 2020; Published: 27 Apr 2020

\section{Food and Hunger}

Food can be defined as "any nutritious substance that people or animals eat or drink, or that plant absorb in order to maintain life and growth is called as food". Cell is the functional unit of our body and each cell of our body in order to function properly requires food. Food provides energy, however there is a limit of energy production in living beings. Since "Food" is described as Prana in Vedas, the minute we are born we need food to sustain life, not only this, the day a female conceives the developing embryo needs food. The type and source of the food varies from developing stage of the embryo to after birth till death. Food (which really includes many types of drinks too - such as milk, juices and cola - but not water) is made up of nutrients. The one who has born on this planet needs food to grow [1]. In other words food is essential for growth and sustenance of life. To some extant every living being is addicted to food, this compulsive behaviour may be in response to an emotion, such as stress, sadness, or anger [2]. Food is a epigenetic factor and influence on gene expression and personality of the person [3, 4]. The human body needs food to function, but food addiction is when a person becomes dependent on certain types of foods only [5]. Our sense organs give clues for the presence of the food which we like, from these clues endocrine glands start working, sending messages through neurones to brain and we feel hungry. Hunger may be 2 types viz (A) True and (B) False.

True: As soon as baby takes birth, it becomes hungry. Each of us is born with an innate sense of hunger. Hunger is the body's physiological need for energy and essential nutrients (carbohydrate, protein, fat, vitamins, minerals, etc.). When the stomach and intestines are empty, they release hormones called ghrelin into the blood and send signals to the hypothalamus $[6,7]$.

This causes particular groups of neurons in the hypothalamus to produce proteins, which make you feel hungry. Ghrelin signals the body to release stomach acids to digest food. If food is not consumed, the stomach acids begin to attack the lining of the stomach, causing hunger pains. You haven't allowed yourself to go without eating for a long enough period of time to have felt true hunger; you may not have experienced it since childhood. For most people these sensations include stomach grumbling, headaches, light-headedness, shakiness, irritability, fatigue, and inability to focus. Since eating removes the symptoms, they are mistakenly believed to be signs of hunger.
False: Unfortunately, many people experience the sensation of hunger for foods rich in energy even though they have ample reserves of energy-containing fat in certain parts of the body and glycogen in the liver. Some people are always "hungry". In order not to be ruled by false hunger (hunger for unnecessary or harmful substances), it is necessary to do two things: learn to recognize the basis of your sensation of hunger and educate your body not to send the wrong messages. False hunger falls into nine basic categories: (a) low blood sugar; (b) an irritation of the lining of the stomach; (c) addictions; (d) the discomfort of the body in utilizing reserves; (e) a desire for stimulation; (f) a genuine need for essential nutrients, expressed by a craving for food dilute in those nutrients; $(\mathrm{g})$ tiredness experienced as a need for food; (h) thinking about, seeing, or smelling food; and (i) habituation to regularity. Hunger for energy-rich, low-nutrition foods when there are excess energy stores in the body is pathological. Arresting and reversing such a condition requires an understanding of its causes. By understanding and then recognizing the following causes of false hunger, you can re-educate yourself to naturally crave only what is needed and nothing else.

\section{Food Addiction}

A false hunger leads to cravings for highly specific food, which in turn makes a person "Food Addict" Food addicts become dependent upon the "good" feelings that are obtained from consuming certain foods, which often perpetuates a continued need to eat, even when not hungry [8-10]. When excess salt or sugar is eliminated by the body, a craving for salt or sugar occurs, not necessarily for salt or sugar per se, but for foods high in salt or sugar. And thus highly palatable foods (which are often rich in fat, sugar, and or salt) trigger chemical reactions in the brain that induce feelings of pleasure and satisfaction. Food addicts become dependent upon the "good" feelings that are obtained from consuming certain foods, which often perpetuates a continued need to eat, even when not hungry $[11,12]$. This can lead to several physicals, emotional, and social consequences, such as digestive issues, heart disease, obesity, low-self-esteem, depression, and isolation. A food addict will often re-engage in these destructive behaviours, even amidst undesired consequences, due to the need for induced feelings of pleasure.

A man or woman may develop an addiction as a result of biological, psychological, or social reasons. Biological causes that may influence the progression of this eating disorder might include hormonal imbalances, abnormalities in various brain structures, side effects from the use of certain medications, or having family members 
with this type of addiction issues. It also might also be the result of psychological factors. Factors included in this category might include emotional or sexual abuse, being a victim or survivor of a traumatic event, having an inability to healthily cope with negative situations, chronic low-self esteem, or experiencing grief or loss. Psychological factors such as these can influence an individual to use food as a coping mechanism to relieve the painful emotions that may have resulted. Lastly, there are social implications that may be involved with food addiction, including factors such as disturbances in family function, pressure from peers or society, social isolation, child abuse, lack of social support, and stressful life events.

\section{Disorders Associated with Food Addiction}

An addiction to food can also be associated with other co-occurring disorders, such as eating disorders or substance abuse. Because food addiction is a complex mental health issue that can have serious complications if left untreated, it is highly recommended that professional help be sought to effectively heal from this disorder. A food addiction can result in many negative physical consequences on the body as an excess of food is consumed. These are some physical effects that may be experienced: Gastro enteric aliments such as Igestive Problems, Malnutrition, Heart disease, Diabetes, Obesity, Chronic pain, reduced sex drive, Arthritis, Kidney/Liver Disease, Osteoporosis [13].

An addiction to food can be debilitating to mental health, especially if there is a lack of support or inadequate help. Some of the nuropsychological effects that may be experienced include: Low selfesteem, Depression, Panic attacks, Increased feelings of anxiety, Feeling sad, hopeless, or in despair, Increased irritability, especially if access to desired food is restricted, Emotional detachment or numbness, Suicidal ideation Stroke Headaches, Lethargy, Chronic fatigue, Sleep disorders. Finally, food addiction can have an impact on your social life and relationships.

\section{Social effects of food addiction include}

Decreased performance at work or school, Isolation from loved ones, Division within family units, Lack of enjoyment in hobbies or activities once enjoyed, Avoidance of social events or functions, Risk of jeopardizing finances or career. However, for many individuals, food addiction can become as serious as drugs are to a substance abuser.

\section{Habituation to Regularity}

How often have you been told of the importance of eating a particular meal at the same time every day? Is it reasonable to assume that a human body, which is never the same from day to day, would always have the same needs at the same time every day? Is the concept of regularity of meals based on physiological needs or on the dictates of the industrialized, assembly-line workplace? If workers were permitted to leave the assembly-line for lunch whenever they wanted to, the effect on production efficiency would be detrimental [14]. Therefore, such workers are required to eat, drink coffee, etc., at the same time. When meals are always at the same time each day, the digestive organs become habituated to begin the digestive process whether or not food is needed or available. Then, if food is not forthcoming, distress, experienced as hunger, ensues. What if your job necessitates eating at the same time every day-hungry or not? Then don't eat if you are not hungry. If hunger later arrives at an inconvenient time, use that opportunity to do without food until the next scheduled meal. You will find that you will lose weight, give your digestive organs a rest, and simultaneously train yourself not to have to eat at the instant you experience hunger.

Dealing with False Hunger: Intermittent Fasting (IF)

The best way to deal with inappropriate cravings is first to experience them. Then attempt to objectively identify them. If sensation of hunger is from an irritated stomach, that knowledge will be very helpful both in dealing with that problem and in quelling the craving. First cultivate the awareness that the hunger is false. Next, develop the tools and resolve to deal with it over the long term. Thus armed, it will be easier to dismiss any thought of eating the wrong food. Just think of how good you feel when you eat properly and are at the correct weight. Think of any health condition with which you may suffer or any excess weight you may be constantly carrying around.

Our human ancestors did not consume three regularly spaced large meals, plus snacks, every day, nor did they live a sedentary life", Human beings have a built-in fight or flight mechanism that helps them to survive without food for some time. Our life style is such that often get used to a certain schedule, especially regarding our dietary habits. Now these habits can be deemed good or bad depending on our perception, beliefs, and goals. One must learn some self control. We simply aren't used to not eating. A feel real hunger will be there if one goes on a 24-hour fast. We will definitely feel hungry and may have a headache or what not because we are simply not used to going this long without food $[15,16]$. Some self-discipline will come in handy at this point. Self-control needs to be a focal point here. The beauty of IF makes our diets simple and allows us to remember what it's actually like to feel satiated and recognize real hunger cues as opposed to the false ones.

The data analyzed showed that "most if not all organ systems respond to intermittent fasting in ways that enable the organism to tolerate or overcome the challenge" and then return to normal [17]. There should be at least 1 non-fasting day between fasting days. During fasting and after fasting food selection and frequency of food intake is important. Focus on whole, natural foods as they tend to fill you up faster than processed, sugary junk food.

\section{References}

1. Gupta PD (2018) Born to die: why do we age? J Cell Tissue Res18.

2. Eisenstein SA, Bischoff AN, Gredysa DM, Antenor-Dorsey JA, Koller JM et al. (2015) Emotional eating phenotype is associated with central dopamine D2 receptor binding independent of body mass index. Sci Rep 5: 11283.

3. Pushkala K, Gupta PD (2013) Epigenetic effect of food for cancer management. Intl J Med Sci Biotech I: 1-7.

4. Sanchez-Roige S, Gray JC, MacKillop J, Chen CH, Palmer AA (2018) The genetics of human personality. Genes Brain Behav 17: 12439.

5. Wilson GT (2010) Eating disorders, obesity and addiction. Eur Eat Disord Rev 18: 341-351.

6. Farooqi IS, Bullmore E, Keogh J, Gillard J, O'Rahilly S, et al. (2007) Leptin regulates striatal regions and human eating behavior. Science 317: 1355.

7. Morris LS, Voon V, Leggio L (2018) Stress, motivation, and the gut-brain axis: a focus on the ghrelin system and alcohol use disorder. Alcohol Clin Exp Res 42: 1378-1389.

8. Hebebrand J, Albayrak O, Adan R, Antel J, Dieguez C, et al. (2014). "Eating addiction", rather than "food addiction", better 
captures addictive-like eating behavior. Neurosci Biobehav Rev 47: 295-306.

9. Schulte EM, Potenza MN, Gearhardt A (2017) A commentary on the "eating addiction" versus "food addiction" perspectives on addictive-like food consumption. Appetite 115: 9-15.

10. Pedram P, Wadden D, Amini P, Gulliver W, Randell E, et al. (2013) Food addiction: its prevalence and significant association with obesity in the general population. PLoS ONE 8: e74832.

11. Finlayson G (2017) Food addiction and obesity: unnecessary medicalization of hedonic overeating. Nat Rev Endocrinol 13: 493-498.

12. Corwin RL (2011) The face of uncertainty eats. Curr Drug Abus Rev 4: 174-181.
13. Wilson GT (2010) Eating disorders, obesity and addiction. Eur Eat Disord Rev 18: 341-351.

14. Gupta PD, Pushkala K (2012 a) Clocks within us LAMBERT Academic Publishing, GmbH \& Co. KG, Saarbrücken, Germany

15. Waheed AA, Gupta PD (1997) Changes in structural and functional properties of rat intestinal brush border membrane during starvation. Life Sci 61: 2425-2433.

16. Gupta A, Gupta PD (2014) Food for Thought in. Nova Science Publishers. C.G.C Press USA.

17. Collier R (2013) Intermittent fasting: the science of going without Canadian Medical Association Journal (CMAJ) 185: E363-E364.
Copyright: (C2020 PD Gupta. This is an open-access article distributed under the terms of the Creative Commons Attribution License, which permits unrestricted use, distribution, and reproduction in any medium, provided the original author and source are credited. 